\title{
ORIGINAL ARTICLE \\ Protective effect of oil from Cornus wilsoniana fruits against carbon tetrachloride-induced hepatic fibrosis in mice
}

\author{
Qiang Liu', Qiang Liu²,3, Xiaohua Lei', Zhenyu Cao', Ju Zhang', Tao Kuang', Guoxing \\ Liu', Yu Fang', Ke Qian', Jie Fu', Huihui Du', Likun Yan', Zhihong Xiao³, Changzhu Li', \\ Xundi Xu'* \\ 'Hunan Provincial Key Laboratory of Hepatobiliary Disease Research \& Division of Hepato-Biliary-Pancreatic Surgery, \\ Department of Surgery, The Second Xiangya Hospital, Central South University, Changsha, China; ${ }^{2}$ College of Life \\ Sciences and Technology, Central South University of Forestry and Technology, Changsha, China; ${ }^{3 H u n a n}$ Provincial \\ key Laboratory of Oils \& Fats Molecular Structure and Function, Hunan Academy of Forestry, Changsha, China
}

Popular scientific summary

- We evaluated the hepatoprotective effect of oil from Cornus Wilsoniana Fruits.

- $\mathrm{CWO}$ protected the liver from damage caused by $\mathrm{CCl}_{4}$ via antioxidant and anti-inflammatory effects.

- We found that CWO can attenuate TGF- $\beta 1 / \mathrm{Smad} 3$ signaling pathway in liver, thereby reducing $\mathrm{CCl}_{4}$-induced hepatic fibrosis in mice.

- Our work is expected to promote $\mathrm{CWO}$ as an edible oil for liver protection.

Abstract

Background: Cornus wilsoniana Wanger is a widely distributed woody oil plant in south China; oil extracted from its fruits has been the main source of edible oil for local residents for hundreds of years. Previous studies have demonstrated that Cornus wilsoniana oil (CWO) has hypolipidemic activity in rats. However, the hepatoprotective effects of CWO and their underlying mechanisms are not clear.

Objective: The purpose of this study was to explore the protective effects and mechanisms of the CWO against carbon tetrachloride $\left(\mathrm{CCl}_{4}\right)$-induced hepatic fibrosis in mice.

Methods: Hepatic fibrosis mouse model was induced by intraperitoneal injection with $1 \mathrm{~mL} / \mathrm{kg} \mathrm{CCl}_{4}$ (mixed $1: 4$ in olive oil) twice a week for 6 weeks. In the meantime, the mice were orally administrated with CWO $(0.5$, $2 \mathrm{~mL} / \mathrm{kg}$ ) once daily for 6 weeks. Serological changes as well as oxidative stress, inflammatory, and histological alteration in the liver were determined.

Results: The results showed that CWO significantly attenuated $\mathrm{CCl}_{4}$-induced serological changes in mice, as assessed by serum markers, including alanine aminotransferase (ALT), aspartate aminotransferase (AST), procollagen III, collagen type IV, hyaluronic acid, and laminin. At the same time, CWO significantly improved $\mathrm{CCl}_{4}$-induced liver histological changes, as detected by hematoxylin and eosin (H\&E), Sirius red, and Masson's trichrome staining. In addition, treatment with CWO reduced oxidative stress and inflammation in the liver. Furthermore, CWO also reduced the expression of extracellular matrix (ECM) in liver induced by $\mathrm{CCl}_{4}$, and TGF- $\beta 1 / \mathrm{Smad} 3$ signaling may be involved in the process.

Conclusions: $\mathrm{CWO}$ ameliorates $\mathrm{CCl}_{4}$-induced hepatic fibrosis by attenuating hepatic oxidative stress, reducing hepatic inflammation and inhibiting TGF- $\beta 1 / \mathrm{Smad} 3$ signaling pathway in liver. CWO may be a potentially beneficial edible oil for the adjuvant treatment of hepatic fibrosis.

Keywords: Cornus wilsoniana oil; hepatic fibrosis; oxidative stress; inflammation; TGF- $\beta 1$

To access the supplementary material, please visit the article landing page 
$\mathrm{H}$ epatic fibrosis is a chronic disease caused by liver injury, and its occurrence and development are complex pathological processes involving multiple factors (1-4). Long-term chronic liver injury stimulates hepatocyte death and regeneration, aggravates hepatic oxidative stress and chronic inflammation, activates hepatic stellate cells (HSCs), and ultimately leads to the deposition of extracellular matrix (ECM), including $\alpha$-smooth muscle actin ( $\alpha$-SMA) and type I collagen (COL1A1) (1, 5-6). Activation of HSCs is a key event in the development of hepatic fibrosis. Due to chronic liver damage, HSCs can be activated, thereby aggravating hepatic fibrosis (7). In addition, aberrant activity of TGF- $\beta 1 / \mathrm{Smad} 3$ signaling pathway is one of the most prominent drivers for the activation and transformation of HSCs into myofibroblasts (8).

Recently, many studies have shown that many natural substances have antifibrotic and liver protective effects (9-11). Cornus wilsoniana Wanger is a widely distributed woody oil plant in south China, which has high fruit yield with high oil content. Because Cornus wilsoniana oil (CWO) contains many beneficial chemicals, such as unsaturated fatty acids (UFAs), phytosterol, alpha tocopherol, and squalene, it has been the main source of edible oil for local residents for hundreds of years. Previous studies have also demonstrated that $\mathrm{CWO}$ has hypolipidemic activity in rats due to its high content of UFAs (12). However, it is still not clear whether CWO can alleviate hepatic fibrosis. Here, we first investigated the role of CWO in hepatic fibrosis and explored its possible mechanisms.

In the present study, we established a carbon tetrachloride $\left(\mathrm{CCl}_{4}\right)$-induced hepatic fibrosis model. To study the role of $\mathrm{CWO}$ in hepatic fibrosis, we treated model mice with different doses of CWO. We found that CWO can significantly improve liver histopathology and serological marker levels, ultimately attenuating the degree of hepatic fibrosis induced by $\mathrm{CCl}_{4}$. The mechanism may be that CWO can improve hepatic oxidative stress, improve hepatic inflammation, and inhibit TGF- $\beta 1 / \mathrm{Smad} 3$ signaling pathway in the liver. Taken together, our studies demonstrated that CWO plays an important role in counteracting the development of $\mathrm{CCl}_{4}$-induced hepatic fibrosis, suggesting that CWO might have the potential to alleviate hepatic fibrosis.

\section{Materials and methods}

\section{Cornus wilsoniana oil}

CWO, supplied by the Hunan Academy of Forestry (Changsha, China), was obtained by subcritical extraction. The fatty acid composition of CWO was determined by gas chromatography-mass spectrometer (GC/ MS). The phytosterol content of CWO was determined by spectrophotometry. The vitamin E and squalene content of CWO were determined by high-performance liquid chromatography.

\section{Reagents}

$\mathrm{CCl}_{4}(\mathrm{C} 112040)$ and olive oil (O108686) were purchased from Aladdin (Aladdin, Shanghai, China). Hematoxylin and eosin (H\&E) staining kit (G1120), Sirius red staining kit (G1471), and Masson's trichrome staining kit (G1340) were all purchased from Solarbio (Solarbio, Beijing, China). TRIzol reagent was purchased from Thermo (Thermo Scientific, MA, USA). Antibodies against $\beta$-actin (60008-1-AP) and F4/80 (28463-1-AP) were purchased from Proteintech (Proteintech, Rosemont, IL, USA). Antibodies against $\alpha$-SMA (ab32575), TGF- $\beta 1$ (ab215715), and p-Smad3 (ab52903) were purchased from Abcam (Abcam, Cambridge, UK). Antibodies against COL1A1 (BA0325) were purchased from Boster (Boster, Beijing, China). A Universal Two-Step Test Kit (PV-9000) was purchased from Zsbio (Zsbio, Beijing, China). Total superoxide dismutase (SOD) (A001-1), malondialdehyde (MDA) (A003-1), and reduced glutathione (GSH) (A0061) test kits were obtained from the Nanjing Jiancheng Biotechnology Institute (Nanjing, China).

\section{Animals and treatment}

C57BL/6J mice were obtained from the Jackson Laboratory (JAX, ME, USA). All mice were housed in well-ventilated sterile cages and provided with food and water $a d$ libitum. After 1 week of adaptation, 24 mice were randomly divided into four groups: a normal control (NC) group that received olive oil, a model group treated with $\mathrm{CCl}_{4}$, a low-dose group (LDG) treated with $\mathrm{CCl}_{4}+\mathrm{CWO}$ $(0.5 \mathrm{~mL} / \mathrm{kg}, \mathrm{IG})$, and a high-dose group (HDG) treated with $\mathrm{CCl}_{4}+\mathrm{CWO}(2 \mathrm{~mL} / \mathrm{kg}, \mathrm{IG})$. When the mice were 6 weeks old, $\mathrm{CCl}_{4}$ was dissolved in olive oil to form a $20 \%$ solution and injected intraperitoneally into mice twice a week at a dose of $5 \mathrm{~mL} / \mathrm{kg}$; the $\mathrm{NC}$ group was injected with the same amount of olive oil. The use of CWO was orally administered daily according to the corresponding dose.

After 6 weeks of continuous administration, the mice were sacrificed $48 \mathrm{~h}$ after the last $\mathrm{CCl}_{4}$ injection without overnight fasting. At sacrifice, mice were anesthetized with diethyl ether; blood and liver samples were collected. Blood samples were centrifuged at 4,000 rpm for $20 \mathrm{~min}$ at $4^{\circ} \mathrm{C}$, and the supernatant was taken for biochemical analysis. The liver was dissected and washed with ice-cold saline. After weighing, the left outer lobe was immersed in $4 \%$ paraformaldehyde, and the remaining liver tissue was immediately frozen in liquid nitrogen and finally transferred to $-80^{\circ} \mathrm{C}$ for long-term storage. The animal experiments were approved by the Animal Care and Use Committee of Central South University. 


\section{Biochemical analysis}

Serum was collected as mentioned above. We measured the serum concentrations of alanine aminotransferase (ALT) and aspartate aminotransferase (AST) using automatic biochemical analyzers (Abbott, Chicago, IL, USA) and then detected the serum levels of procollagen III (PC III), collagen type IV (IV-C), hyaluronic acid (HA), and laminin (LN) with radioimmunoassay kits (Yuande Bio-Medicine, Beijing, China). To evaluate oxidative stress, we used commercial kits to detect SOD, GSH, and MDA levels in liver homogenates according to the manufacturer's protocols.

\section{Histopathology}

Liver tissues fixed in 4\% paraformaldehyde were embedded in paraffin and then cut into $4-\mu \mathrm{m}$-thick sections. To quantify the hepatic fibrosis area, these sections were prepared for staining with H\&E, Sirius red, and Masson's trichrome. For Sirius red collagen staining, thin sections were deparaffinized according to standard procedures and stained with Sirius red staining solution for $1 \mathrm{~h}$ at room temperature. After washing with water, the sections were gradually dehydrated in ethanol and then sealed with neutral balsam. For Masson's trichrome staining, the sections were routinely deparaffinized and hydrated and then stained in steps using Masson's trichrome kit. The severity of hepatic fibrosis was assessed under light microscopy (Olympus, Hamburg, Germany). Images were obtained of random fields, and representative views of liver sections are shown.

\section{Immunohistochemistry}

The expression of $\alpha$-SMA, COL1A1, and F4/80 in the liver was observed by immunohistochemical staining, as described below. Briefly, the tissue sections were deparaffinized and hydrated, incubated with $0.3 \%$ hydrogen peroxide to block endogenous peroxidase, subjected to antigen repair with citrate at high pressure, and finally incubated at $37^{\circ} \mathrm{C}$ with $\alpha$-SMA (1:500 dilution), COL1A1 (1:500 dilution), and F4/80 (1:1000 dilution) antibodies for $1 \mathrm{~h}$. After the sections were incubated with the primary antibodies, they were processed using a Universal Two-Step Test Kit. Thereafter, the sections were counterstained with hematoxylin and sealed with neutral balsam.

\section{Real-Time Quantitative Polymerase Chain Reaction (RT-qPCR)}

Approximately $20 \mathrm{mg}$ of liver tissue was homogenized in $1 \mathrm{~mL}$ of TRIzol reagent. Total RNA was extracted according to the manufacturer's instructions. The entire experimental procedure was carried out according to the instructions; mRNA expression fold changes were analysis by the relative quantification method $\left(2^{-\Delta \Delta C t}\right)$. The following primer pairs were used: IL-6, (forward)

$\begin{array}{lll}\text { 5'-GACTTCCATCCAGTTGCCTT-3' } & \text { and } & \text { (reverse) } \\ \text { 5'-ATGTGTAATTAAGCCTCCGACT-3'; } & \text { IL-I } \beta, & \text { (forward) } \\ \text { 5'-TGAAATGCCACCTTTTGACAGT-3', } & \text { and } & \text { (reverse) } \\ \text { 5'-TTCTCCACAGCCACAATGAGT-3'; } & \text { TNF } \alpha, & \text { (forward) } \\ \text { 5'-AGCACAGAAAGCATGATCCG-3' } & \text { and } & \text { (reverse) } \\ \text { 5'-CACCCCGAAGTTCAGTAGACA-3'; } & \text { MCP-I, } & \text { (forward) } \\ \text { 5'-AGCAGCAGGTGTCCCAAA-3' } & \text { and } & \text { (reverse) } \\ \text { 5'-CTGAAGACCTTAGGGCAGAT-3'; } & \beta \text {-actin, } & \text { (forward) } \\ \text { 5'-ACATCCGTAAAGACCTCTATGCC-3', } & \text { and } & \text { (reverse) } \\ \text { 5'-TACTCCTGCTTGCTGATCCAC-3'. } & & \end{array}$

\section{Western blotting}

Liver tissue total protein was extracted with radioimmunoprecipitation assay (RIPA) buffer, and the concentration was determined by the Bicinchoninic Acid (BCA) method. Thirty micrograms of liver protein lysate was electrophoresed on $10 \%$ sodium dodecyl sulphate polyacrylamide gel electrophoresis (SDS-PAGE). The separated proteins were transferred onto polyvinylidene fluoride (PVDF) membranes, which were blocked with 5\% skim milk powder for $1 \mathrm{~h}$ at room temperature. Then, the membranes were incubated overnight at $4^{\circ} \mathrm{C}$ with $\alpha$-SMA (1:1,000 dilutions), COL1A1 (1:500 dilutions), TGF- $\beta 1$ (1:1,000 dilutions), and $\mathrm{p}-\operatorname{Smad} 3$ (1:2,000 dilutions) primary antibodies. $\beta$-Actin (1:5,000 dilution) was used as a control. After washing with TBST, the membranes were incubated with secondary antibodies (1:5,000 dilution) for $1 \mathrm{~h}$ at room temperature, then detected with enhanced chemiluminescence system. Images were captured, and Image $\mathbf{J}$ software was used to analyze the grey values of the target protein bands.

\section{Statistical analyses}

The data are expressed as the mean \pm standard deviation (SD) and were analyzed using SPSS 19.0 software. Except pathological data, all other experimental data were analyzed by one-way analysis of variance (ANOVA) followed by Tukey multiple comparison test. The pathological data of the liver were analyzed by the Kruskal-Wallis nonparametric test, followed by a Mann-Whitney $U$-test. $P<0.05$ was taken to indicate that a difference was statistically significant. Histograms were generated using GraphPad Prism 7 software (San Diego, CA, USA).

\section{Results}

Chemical composition and physicochemical properties of CWO CWO was qualitatively and quantitatively characterized by $\mathrm{GC} / \mathrm{MS}$, revealing a total of six major chemical constituents; the results of GC/MS analysis of fatty acid methyl esters are shown in Fig. 1 and Table 1. In addition to fatty acid components, we also found some other 


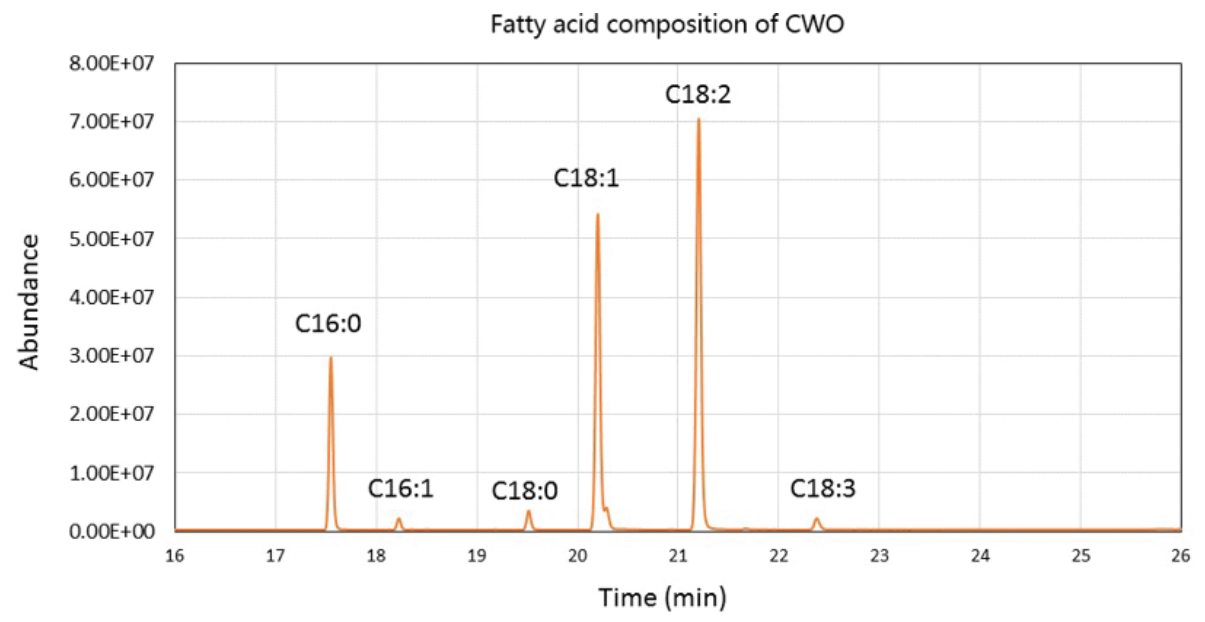

Fig. 1. Fatty acid composition of CWO. The fatty acid composition of CWO was detected by gas chromatography-mass spectrometer.

Table 1. Relative content of fatty acids in Cornus wilsoniana oil (CWO)

\begin{tabular}{llc}
\hline No. & Fatty acid & Relative \% \\
\hline 1 & CI6:0 (Palmitic) & 15.48 \\
2 & CI6:I (Palmitoleic) & 1.10 \\
3 & CI8:0 (Stearic) & 1.89 \\
4 & CI8:I (Oleic) & 35.71 \\
5 & CI8:2 (Linoleic) & 44.49 \\
6 & CI8:3 (Linolenic) & 1.32 \\
& Saturated fatty acids & 17.37 \\
& Monounsaturated fatty acids & 36.81 \\
& Polyunsaturated fatty acids & 45.81 \\
\hline
\end{tabular}

Table 2. Other beneficial factors in Cornus wilsoniana oil (CWO)

\begin{tabular}{lll}
\hline No. & Compound & Content \\
\hline $\mathrm{I}$ & Phytosterol & $1.98 \mathrm{mg} / \mathrm{g}$ \\
2 & Vitamin E (alpha tocopherol) & $0.60556 \mathrm{mg} / \mathrm{g}$ \\
3 & Squalene & $0.0324 \mathrm{~g} / \mathrm{kg}$ \\
\hline
\end{tabular}

beneficial factors in CWO, including phytosterols, vitamin E (alpha tocopherol), and squalene (Table 2). The physicochemical properties of CWO (acid value, $5.31 \mathrm{mg}$ $\mathrm{KOH} / \mathrm{g}$; iodine value, $104.2 \mathrm{~g} / 100 \mathrm{~g}$; saponification value, $200.89 \mathrm{mg} \mathrm{KOH} / \mathrm{g}$ ) are similar with other edible oils, such as peanut oil and soybean oil.

\section{CWO attenuated $\mathrm{CCl}_{4}$-induced liver injury}

To test the effect of CWO on hepatic fibrosis, we used a $\mathrm{CCl}_{4}$-induced hepatic fibrosis model in this study. The $\mathrm{CCl}_{4}$-induced mouse hepatic fibrosis model largely mimicked human hepatic fibrosis. Gross examination showed that pathological changes, such as small nodules on the surface of the liver, occurred in the livers of mice injected with $\mathrm{CCl}_{4}$ compared with those of mice injected with olive oil, but CWO treatment attenuated pathological changes in the liver. As shown in Fig. 2a, H\&E staining showed that the model group had pseudolobule formation in the liver and inflammatory cell infiltration in the portal area, while CWO reduced inflammatory cell infiltration and pseudolobule formation, especially in the HDG. The liver/body weight ratio of the model group was significantly $(P<0.01)$ higher than that of the NC group; however, CWO could significantly $(P<0.05)$ reduce the ratio in HDG (Fig. $2 \mathrm{~b}$ and Supplementary Table S1). ALT and AST are serological markers of liver damage, and $\mathrm{CCl}_{4}$ treatment resulted in significant $(P<0.01)$ increases in ALT and AST, whereas CWO effectively $(P<0.01)$ reduced the levels of these markers (Fig. 2c). Other serum biochemical parameters, including PC III, IV-C, HA, and LN, are important markers of the severity of hepatic fibrosis. In the model group, the levels of serum PC III were significantly $(P<0.01)$ increased compared with those in the $\mathrm{NC}$ group. Compared with $\mathrm{CCl}_{4}$ treatment alone, treatment with $\mathrm{CWO}$ along with $\mathrm{CCl}_{4}$ significantly $(P<$ 0.05 ) decreased the serum levels of precollagen type III in a dose-dependent manner (Fig. 2d). Similar results were observed for other hepatic fibrosis serological markers, such as IV-C, HA, and LN (Fig. 2d).

\section{CWO improved $\mathrm{CCl}_{4}$-induced histopathological alterations}

To assess the effect of $\mathrm{CWO}$ on $\mathrm{CCl}_{4}$-induced hepatic fibrosis, Sirius red staining and Masson's trichrome were used to quantify the area of hepatic fibrosis. In the NC group, the liver had a normal lobular structure with a central vein and radial hepatic cord, whereas $\mathrm{CCl}_{4}$ treatment resulted in severe vacuolar degeneration of hepatocytes, inflammatory cell infiltration, impairment of lobular structure, large fibrous septum formation, pseudolobule formation, and collagen fiber deposition. However, CWO 


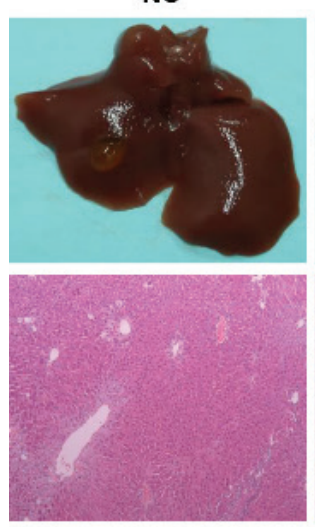

C
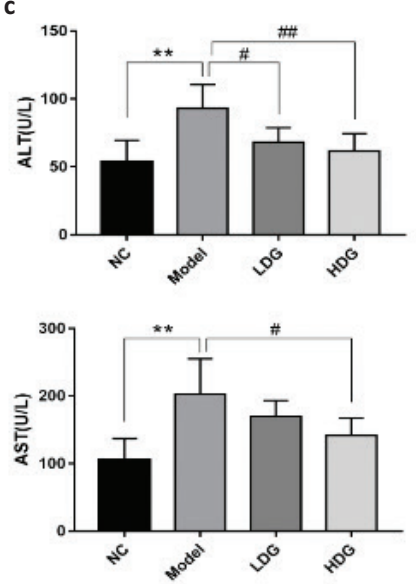

LDG
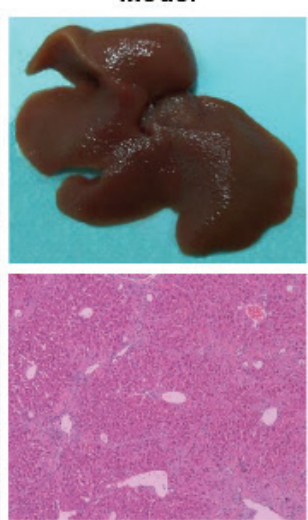

.
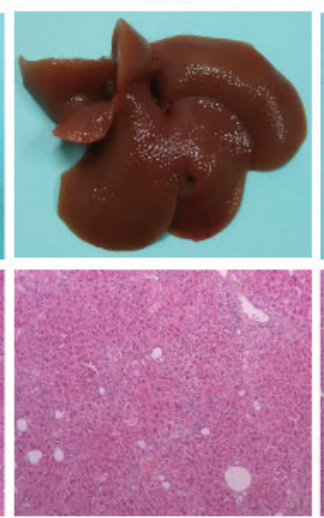

b

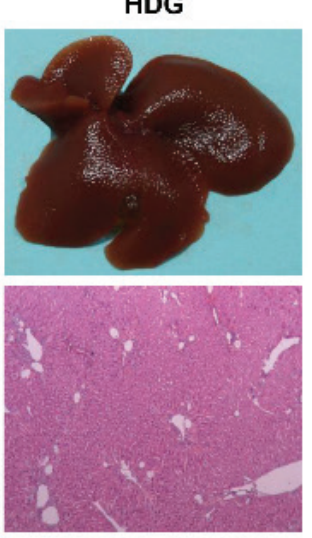

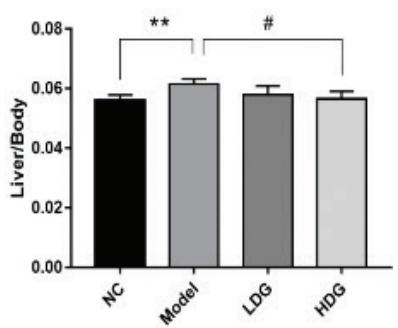

d
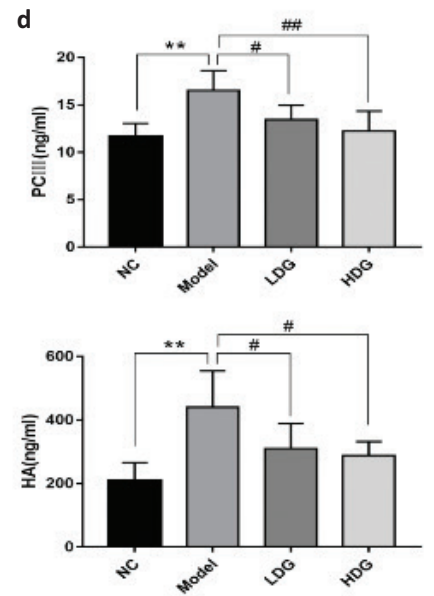
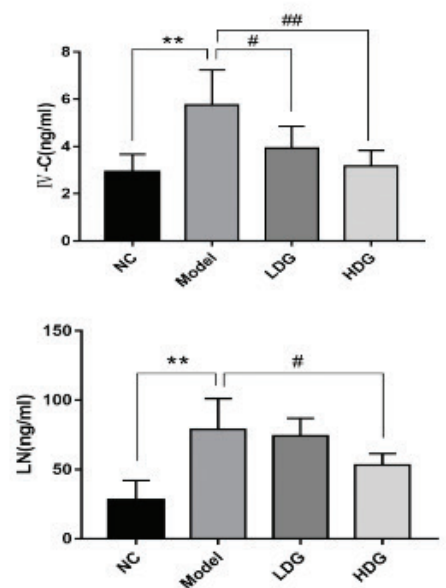

Fig. 2. CWO attenuated $\mathrm{CCl}_{4}$-induced liver injury. (a) The morphology of whole livers and H\&E-stained liver sections (100×) from the four groups (normal control group: olive oil treatment; model group: $\mathrm{CCl}_{4}$ treatment; low-dose group: $\mathrm{CCl}_{4}+\mathrm{CWO}$ $(0.5 \mathrm{~mL} / \mathrm{kg})$ treatment; high-dose group: $\mathrm{CCl}_{4}+\mathrm{CWO}(2 \mathrm{~mL} / \mathrm{kg})$ treatment). Representative photographs are shown. (b) The liver-to-body weight ratios of the indicated four groups. (c) ALT and AST levels in serum derived from the mice in the indicated four groups were determined with standard enzymatic assay kits. (d) Serum levels of PC III, IV-C, HA, and LN were detected using commercially available kits. The data are expressed as the mean $\pm \operatorname{SD}(n=6 /$ group $) ; * * P<0.01, \# P<0.05, \# \# P<0.01$.

significantly attenuated the series of liver histopathological changes caused by $\mathrm{CCl}_{4}$, especially in the $\mathrm{HDG}$ (Fig. 3a and Table 3).

CWO inhibited oxidative stress caused by $\mathrm{CCl}_{4}$

Oxidative stress is an important factor in the process of hepatic fibrosis, and excessive oxidative stress can exacerbate hepatic fibrosis. Liver oxidative stress can be evaluated by measuring liver tissue metrics, including SOD, GSH, and MDA levels. In this study, we hypothesized that $\mathrm{CWO}$ might attenuate $\mathrm{CCl}_{4}$-induced liver injury by inhibiting oxidative stress. Compared with olive oil treatment alone, $\mathrm{CCl}_{4}$ treatment significantly $(P<0.01)$ reduced SOD and GSH levels in liver tissue. However, treatment with high-dose CWO significantly $(P<0.05)$ reversed $\mathrm{CCl}_{4}$-induced SOD and GSH depletion (Fig. 4a and b). In contrast, compared with olive oil treatment alone, $\mathrm{CCl}_{4}$ treatment significantly $(P<0.01)$ increased the levels of MDA, while high-dose
CWO treatment markedly $(P<0.05)$ reduced MDA levels (Fig. 4c).

\section{CWO attenuated CCl4-induced inflammatory response}

Inflammatory response is an important process in the development of hepatic fibrosis. $\mathrm{CCl}_{4}$ treatment can aggravate the inflammatory response in mouse liver. In this study, we assumed that $\mathrm{CWO}$ treatment could reduce $\mathrm{CCl}_{4}$-induced liver inflammation. As shown in Fig. 5a, compared with the $\mathrm{NC}$ group, $\mathrm{CCl}_{4}$ treatment significantly increased the number of $\mathrm{F} 4 / 80$ positive cells in the liver. However, concurrent administration of CWO markedly reduced the number of $\mathrm{F} 4 / 80$ positive cells. Similarly, $\mathrm{CCl}_{4}$ treatment significantly $(P<0.01)$ increased the transcript levels of IL-6, IL-1 $\beta$, TNF $\alpha$, and monocyte chemoattractant protein-1 (MCP-1) in the liver; concurrent administration of CWO markedly $(P<0.05)$ reduced their transcript levels in the liver compared with $\mathrm{CCl}_{4}$ administration alone in a dose-dependent manner. 

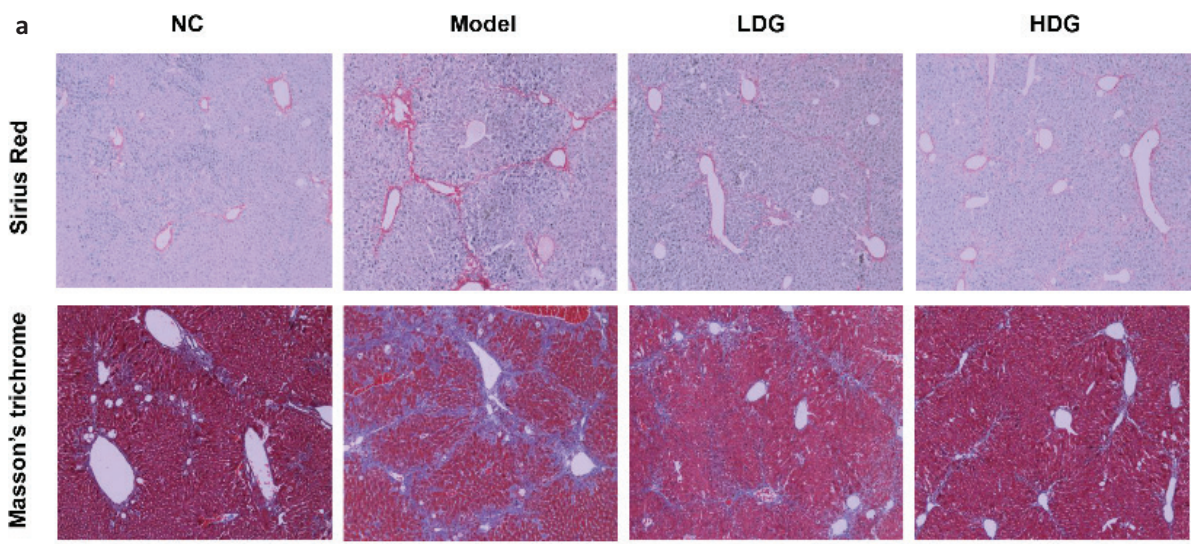

b
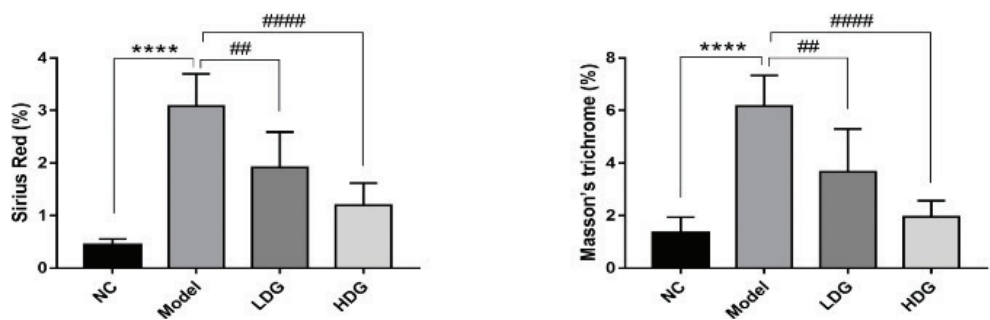

Fig. 3. CWO suppressed hepatic fibrogenesis in a $\mathrm{CCl}_{4}$-induced mouse model. (a) Representative histology of Sirius red, Masson's trichrome are shown $(100 \times)$. (b) Quantification of positive staining areas was measured by Image J software. The data are

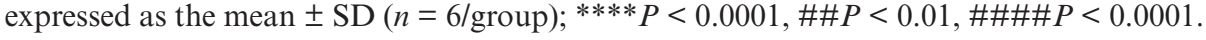

Table 3. Degree of hepatic fibrosis in each group

\begin{tabular}{|c|c|c|c|c|c|c|c|c|}
\hline \multirow[t]{2}{*}{ Group } & \multirow[t]{2}{*}{$n$} & \multicolumn{7}{|c|}{ Pathological grading of hepatic fibrosis } \\
\hline & & 0 & I & II & III & IV & V & $\mathrm{VI}$ \\
\hline Normal control (NC) & 6 & 6 & 0 & 0 & 0 & 0 & 0 & 0 \\
\hline Model & 6 & 0 & 0 & 0 & 2 & 3 & 1 & 0 \\
\hline Low-dose group (LDG) & 6 & 0 & I & 3 & 2 & 0 & 0 & 0 \\
\hline High-dose group (HDG) & 6 & 0 & 3 & 2 & 1 & 0 & 0 & 0 \\
\hline
\end{tabular}

Data are presented as the mean of 10 fields. $n$, Number of mice.

aSignificant difference versus NC group $(P<0.0 \mathrm{I})$.

bignificant difference versus model group $(P<0.01)$.

'Significant difference versus model group $(P<0.01)$.
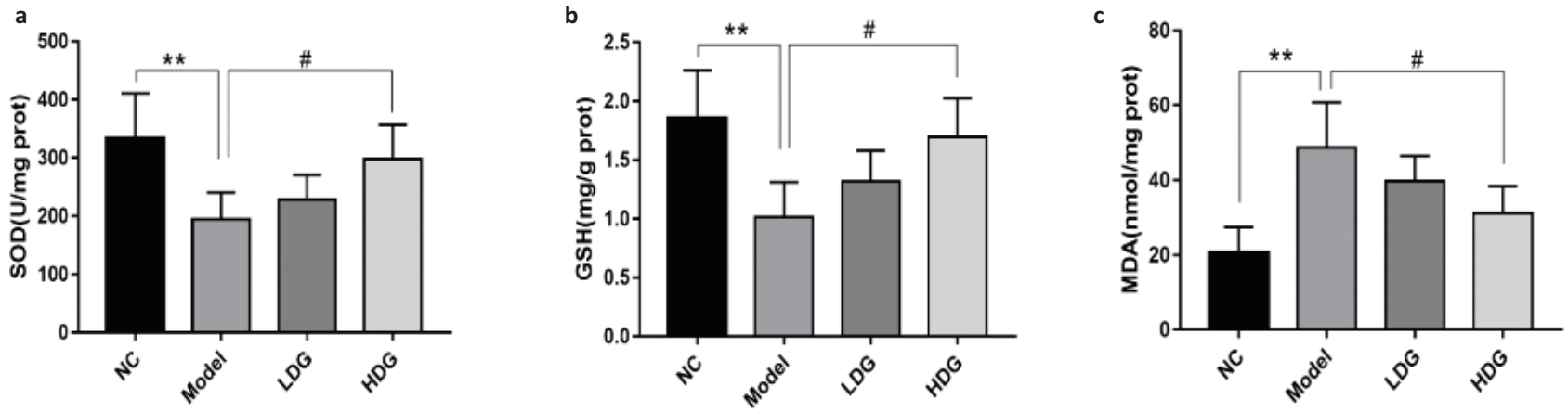

Fig. 4. CWO attenuated oxidative stress in a $\mathrm{CCl}_{4}$-induced mouse model. (a-c) The content of SOD, GSH, and MDA in the liver was detected using commercially available kits. The data are presented as the mean $\pm \mathrm{SD}(n=6 / \mathrm{group})$; $* * P<$ $0.01, \# P<0.05$. 
NC

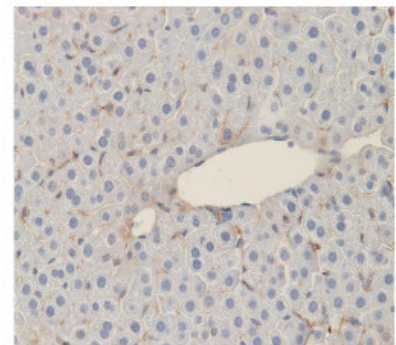

Model

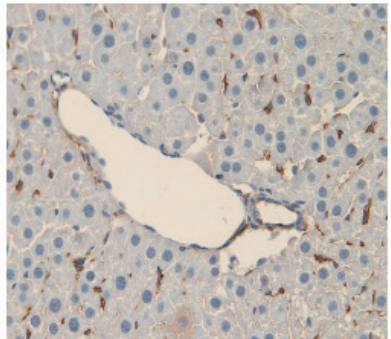

LDG

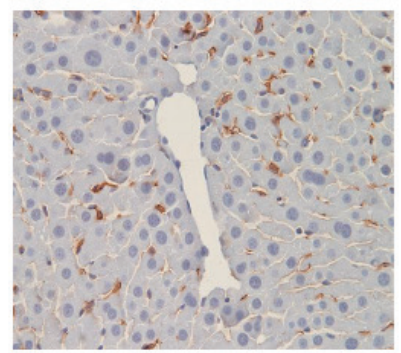

HDG

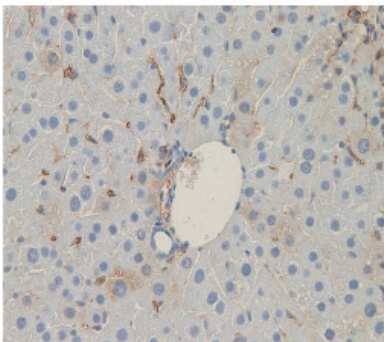

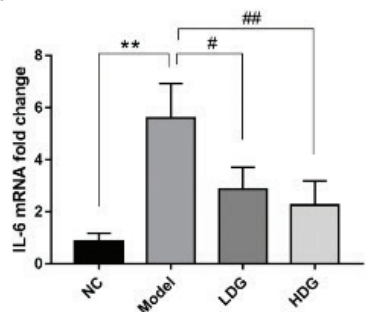

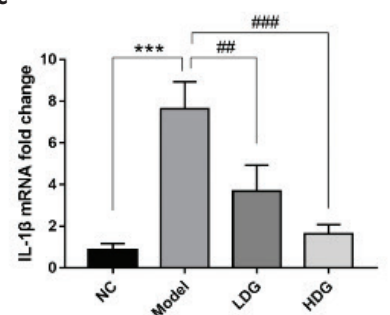

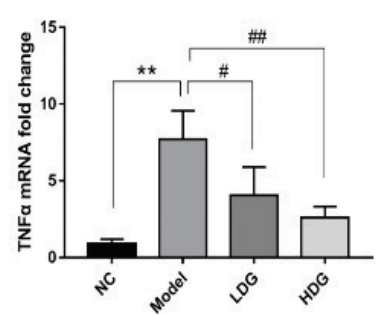

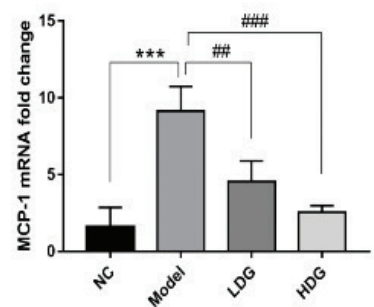

Fig. 5. Effect of CWO on proinflammatory cytokines and chemokines. (a) The expression of F4/80 in the liver tissues was determined using immunohistochemistry assays (200×). (b) The transcript levels of IL-6, IL-1 $\beta$, TNFo, and MCP-1 in the liver were determined using RT-qPCR assays. The data are expressed as the mean $\pm \mathrm{SD}(n=6 /$ group $) ; * * P<0.01,{ }^{* * *} P<0.001, \# P$ $<0.05, \# \# P<0.01, \# \# \# P<0.001$.
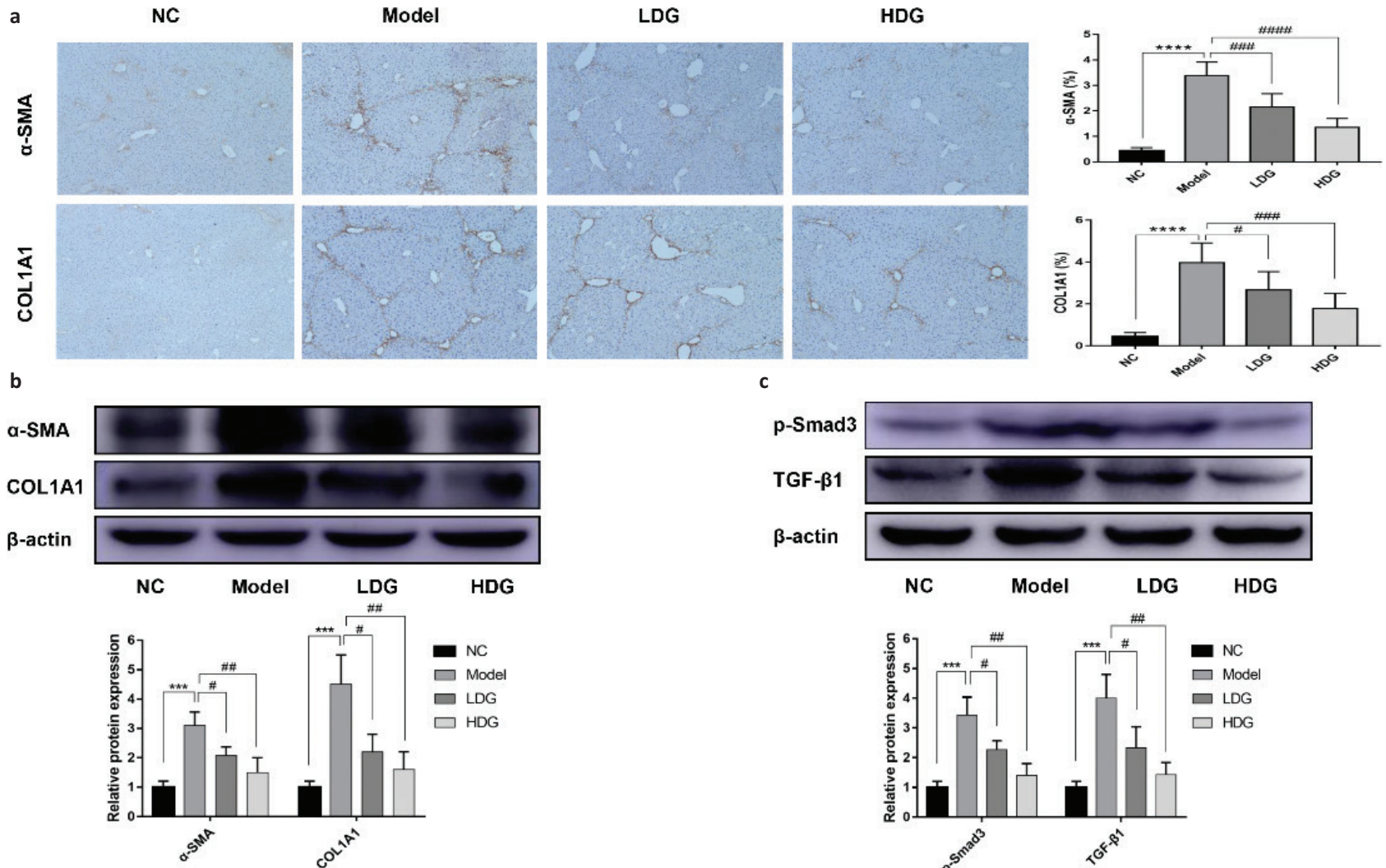

c
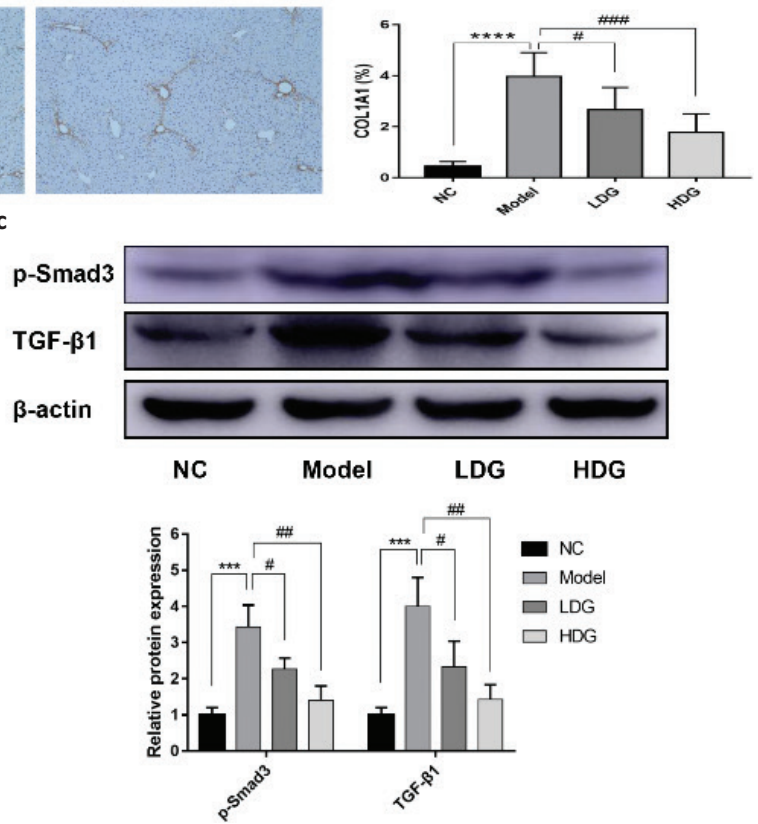

Fig. 6. CWO reduced the expression of ECM and inhibited TGF- $\beta 1 /$ Smad3 signaling pathway. (a) The expression of $\alpha$-SMA and COL1A1 in the liver tissues was determined using immunohistochemistry assays (200×). Quantification of positive staining areas was measured by Image J software. (b) The protein levels of $\alpha$-SMA and COL1A 1 in the liver tissues of the four groups of mice were determined using Western blot assays. (c) The protein levels of TGF- $\beta 1$ and p-Smad 3 in the liver tissues of the four groups of mice were determined

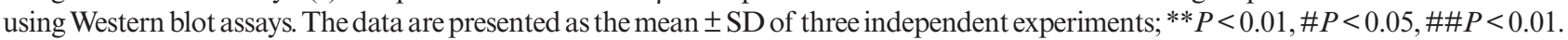




\section{Effect of CWO on ECM and TGF- $\beta$ I/Smad3 signaling pathway} $\alpha$-SMA and COL1A1 are the main ECM proteins and secreted by activated HSCs, and their expression increases the production of chemokines that can recruit various inflammatory cells, thereby further aggravating hepatic fibrosis. Immunohistochemical staining of $\alpha$-SMA and COL1A1 was used to evaluate ECM deposition. As shown in Fig. 6a, the expression of $\alpha$-SMA and COL1A1 was observed in smooth muscle cells of blood vessels in the $\mathrm{NC}$ group. In the CCl4-induced model group, $\alpha$-SMA and COL1A1 expression was significantly elevated in the portal areas and collagen fiber deposition areas. In contrast, the expression of $\alpha$-SMA and COL1A1 protein in the CWO treatment group was significantly lower than that in the CCl4-induced model group. Western blot assays was also used to detect the expression of $\alpha$-SMA and COL1A1. The expression of $\alpha$-SMA and COL1A1 was greatly $(P<0.001)$ enhanced by $\mathrm{CCl}_{4}$ treatment. However, concurrent administration of CWO markedly $(P<0.05)$ decreased $\alpha$-SMA and COL1A1 expression compared with $\mathrm{CCl}_{4}$ administration alone in a dose-dependent manner (Fig. 6b). TGF- $\beta 1 / \mathrm{Smad} 3$ signaling pathway is one of the most important pathways to activate HSCs and promote hepatic fibrosis. In the Western blot assays, we found that expression of Transforming Growth Factor- $\beta 1$ (TGF$\beta 1)$ and $\mathrm{p}-\mathrm{Smad} 3$ was markedly $(P<0.001)$ enhanced by $\mathrm{CCl}_{4}$ treatment compared with $\mathrm{NC}$ group, while concurrent treatment with CWO largely $(P<0.05)$ restored the levels of TGF- $\beta 1$ and $\mathrm{p}-\mathrm{Smad} 3$, especially in the HDG (Fig. 6c).

\section{Discussion}

In the present study, we investigated the efficacy of oral $\mathrm{CWO}$ for treatment of $\mathrm{CCl}_{4}$-induced hepatic fibrosis in mice. Through a 6-week in vivo experiment, our research demonstrated that supplementary CWO was effective in attenuating liver injury and hepatic fibrosis, suggesting that CWO has potential value for the prevention and treatment of hepatic fibrosis. Our experiments further suggested that the effectiveness of this treatment may be attributable to its antioxidant capacity, anti-inflammatory effect and its effects on inhibiting TGF- $\beta 1 /$ Smad 3 signaling pathway.

Serological markers are important indicators for assessing liver injury and hepatic fibrosis. Plasma ALT and AST are sensitive indicators of liver damage. HA, PC III, IV-C, and $\mathrm{LN}$ are markers that reflect the severity of hepatic fibrosis (13-15). To confirm the hepatoprotective effect of CWO, we detected these serological markers to assess liver function and the degree of hepatic fibrosis. We found that CWO reduced ALT and AST levels in a dose-dependent manner and also improved the levels of serum markers of hepatic fibrosis, including HA, PC III, IV-C, and LN.
These results indicated that $\mathrm{CWO}$ has a hepatoprotective effect and can alleviate liver injury and hepatic fibrosis.

Histopathological analysis is used as a direct means of evaluating the protective effects of CWO. Hepatic fibrosis histopathology is characterized by hepatocyte structural disorder, extensive hepatic steatosis, balloon-like changes, inflammatory necrosis, collagen deposition, and diffuse fibrous septum formation (16-17). In the present study, the H\&E, Sirius red, and Masson's trichrome results showed that CWO can reduce liver inflammation, inhibit collagen fiber deposition, and improve liver tissue structure. The results of immunohistochemical staining of $\alpha$-SMA and COL1A1 further confirmed that CWO can alleviate the deposition of collagen fibers and the formation of pseudolobules, indicating that CWO can ameliorate liver histopathological changes.

Regarding the mechanism of hepatic fibrosis, a growing number of studies have shown that oxidative stress contributes to the formation of hepatic fibrosis and that abnormal oxidative stress conditions facilitate the activation of various signaling pathways, thereby causing lipid peroxidation, activating HSCs, and accelerating the development of hepatic fibrosis (18-20). SOD and GSH are important antioxidants in organisms; they can inhibit free radical-initiated lipid peroxidation and protect against free radical damage to body tissues (21-22). Their content in liver tissue can reflect the antioxidant capacity of the liver. MDA is one of the most important products of lipid peroxidation; it causes cross-linking of macromolecules such as proteins and nucleic acids, and it can denature biofilms and produce cytotoxicity (2122). Its liver content can also reflect the oxidative stress state of the liver. In the present study, the liver content of SOD and $\mathrm{GSH}$ was decreased in the $\mathrm{CCl}_{4}$-treated model group compared with the $\mathrm{NC}$ group, whereas the liver content of MDA was increased. However, CWO treatment could reverse this trend and reduce hepatic oxidative stress caused by $\mathrm{CCl}_{4}$. The mechanism may be related to the fact that CWO is rich in UFAs and other antioxidant components, including phytosterol, alpha tocopherol, and squalene. These data suggest that CWO exerts a protective effect against hepatic fibrosis by inhibiting oxidative stress.

Liver inflammation is another important mechanism for the development of hepatic fibrosis (23-24). Chronic liver inflammation is often accompanied by the upregulation of proinflammatory cytokines and chemokines, which can aggravate hepatic fibrosis (23-24). IL-6, IL-1 $\beta$, and TNF $\alpha$ are important proinflammatory cytokines; MCP-1 is a major chemokine; and F4/80 is a marker of mouse macrophages. IL- 6 and IL- $1 \beta$ can induce hepatic inflammation and ECM synthesis in liver (25). TNF $\alpha$ aggravates the development of hepatic fibrosis caused by various factors (26-28). MCP-1 has 
chemotactic activity for monocytes and can activate monocytes and macrophages (29). In this study, administration of CWO could reduce the number of F4/80 positive cells in liver, and inhibit the transcript levels of IL-6, IL-1 $\beta$, TNF $\alpha$, and MCP-1 in the liver. These results indicate that $\mathrm{CWO}$ can alleviate hepatic fibrosis by inhibiting liver inflammation.

In addition to the above mechanisms leading to hepatic fibrosis, activation of HSCs is another one of the most widely accepted mechanisms. Activation of HSCs is an important event in the process of hepatic fibrosis, and activated HSCs transform into a myofibroblast-like phenotype, after which they secrete ECM proteins such as $\alpha$-SMA and COL1A1 (30-32). Therefore, the presence of $\alpha$-SMA and COL1A1 is often considered as HSC activity markers during the progression of hepatic fibrosis. TGF- $\beta 1$, a pleiotropic cytokine, plays a key role in the development of hepatic fibrosis and can activate Smad3 to accelerate fibrosis progression. Many studies have indicated that TGF- $\beta 1 / \mathrm{Smad} 3$ signaling pathway is an important pathway to promote the activation of HSCs and the production of ECM (33-35). In the present study, our results showed that $\mathrm{CCl}_{4}$ treatment could activate TGF- $\beta 1 /$ Smad3 signaling pathway and increase ECM expression in liver tissue, while concurrent administration of CWO could inhibit the TGF- $\beta 1 / \mathrm{Smad} 3$ signaling pathway and reduce ECM expression in a dose-dependent manner. Therefore, these data suggest that the key protective effect of CWO against hepatic fibrosis may be mediated by inhibition the activation of TGF- $\beta 1 / \mathrm{Smad} 3$ signaling pathway.

\section{Conclusion}

In summary, we have demonstrated for the first time that $\mathrm{CWO}$ can ameliorate $\mathrm{CCl}_{4}$-induced hepatic fibrosis by attenuating hepatic oxidative stress, reducing hepatic inflammation, and inhibiting TGF- $\beta 1 / \mathrm{Smad} 3$ signaling pathway in liver. Our findings indicate that CWO may be a potentially beneficial edible oil for the adjuvant treatment of hepatic fibrosis.

\section{Acknowledgments}

This work was supported by the National Natural Science Foundation of China (grant number 81670111).

\section{Conflicts of interest and funding}

The authors declare no potential conflicts of interest. The authors have not received any funding or benefits from industry or elsewhere to conduct this study except those mentioned under the Acknowledgements section.

\section{References}

1. Bataller R, Brenner DA. Liver fibrosis. J Clin Invest 2005; 115 : 209-18. doi: 10.1172/JCI24282
2. Lim YS, Kim WR. The global impact of hepatic fibrosis and end-stage liver disease. Clin Liver Dis 2008; 12: 733-46. doi: 10.1016/j.cld.2008.07.007

3. Lackner C, Tiniakos D. Fibrosis and alcohol-related liver disease. J Hepatol 2019; 70: 294-304. doi: 10.1016/j.jhep.2018.12.003

4. Seitz HK, Bataller R, Cortez-Pinto H, Gao B, Gual A, Lackner C, et al. Alcoholic liver disease. Nat Rev Dis Primers 2018; 4: 16. doi: 10.1038/s41572-018-0014-7

5. Trautwein C, Friedman SL, Schuppan D, Pinzani M. Hepatic fibrosis: concept to treatment. J Hepatol 2015; 62: S15-24. doi: 10.1016/j.jhep.2015.02.039

6. Tsuchida T, Friedman SL. Mechanisms of hepatic stellate cell activation. Nat Rev Gastroenterol Hepatol 2017; 14: 397-411. doi: $10.1038 /$ nrgastro. 2017.38

7. Friedman SL. Hepatic stellate cells: protean, multifunctional, and enigmatic cells of the liver. Physiol Rev 2008; 88: 125-72. doi: $10.1152 /$ physrev.00013.2007

8. Iwaisako K, Jiang C, Zhang M, Cong M, Moore-Morris TJ, Park TJ, et al. Origin of myofibroblasts in the fibrotic liver in mice. Proc Natl Acad Sci U S A 2014; 111: E3297-305. doi: 10.1073/pnas. 1400062111

9. Narayanankutty A, Palliyil DM, Kuruvilla K, Raghavamenon AC. Virgin coconut oil reverses hepatic steatosis by restoring redox homeostasis and lipid metabolism in male Wistar rats. $\mathrm{J}$ Sci Food Agric 2018; 98: 1757-64. doi: 10.1002/jsfa.8650

10. Ogaly HA, Eltablawy NA, Abd-Elsalam RM. Antifibrogenic influence of Mentha piperita L. essential oil against CCl4-induced liver fibrosis in rats. Oxid Med Cell Longev 2018; 2018: 1-15. doi: $10.1155 / 2018 / 4039753$

11. Tian Z, Jia H, Jin Y, Wang M, Kou J, Wang C, et al. Chrysanthemum extract attenuates hepatotoxicity via inhibiting oxidative stress in vivo and in vitro. Food Nutr Res 2019; 63. doi: 10.29219/fnr.v63.1667

12. Fu J, Zhang XW, Liu K, Li QS, Zhang LR, Yang XH, et al. Hypolipidemic activity in Sprague-Dawley rats and constituents of a novel natural vegetable oil from Cornus wilsoniana fruits. J Food Sci 2012; 77: H160-9. doi: 10.1111/j.1750-3841.2012.02786.x

13. Neuman MG, Cohen LB, Nanau RM. Hyaluronic acid as a non-invasive biomarker of liver fibrosis. Clin Biochem 2016; 49: 302-15. doi: 10.1016/j.clinbiochem.2015.07.019

14. Gudowska M, Cylwik B, Chrostek L. The role of serum hyaluronic acid determination in the diagnosis of liver fibrosis. Acta Biochim Pol 2017; 64: 451-7. doi: 10.18388/abp.2016_1443

15. Parsian H, Rahimipour A, Nouri M, Somi MH, Qujeq D, Fard MK, et al. Serum hyaluronic acid and laminin as biomarkers in liver fibrosis. J Gastrointestin Liver Dis 2010; 19: 169-74.

16. Doi K, Kurabe S, Shimazu N, Inagaki M. Systemic histopathology of rats with CCl4-induced hepatic cirrhosis. Lab Anim 1991; 25: 21-5. doi: 10.1258/002367791780808121

17. Lee YA, Wallace MC, Friedman SL. Pathobiology of liver fibrosis: a translational success story. Gut 2015; 64: 830-41. doi: 10.1136/gutjnl-2014-306842

18. Lee CP, Shih PH, Hsu CL, Yen GC. Hepatoprotection of tea seed oil (Camellia oleifera Abel.) against CCl4-induced oxidative damage in rats. Food Chem Toxicol 2007; 45: 888-95. doi: 10.1016/j.fct.2006.11.007

19. Li S, Tan HY, Wang N, Zhang ZJ, Lao L, Wong CW, et al. The role of oxidative stress and antioxidants in liver diseases. Int $\mathrm{J}$ Mol Sci 2015; 16: 26087-124. doi: 10.3390/ijms161125942

20. Noori M, Jafari B, Hekmatdoost A. Pomegranate juice prevents development of non-alcoholic fatty liver disease in rats by attenuating oxidative stress and inflammation. J Sci Food Agric 2017; 97: 2327-32. doi: 10.1002/jsfa.8042 
21. Durasevic S, Jasnic N, Prokic M, Grigorov I, Martinovic V, Dordevic J, et al. The protective role of virgin coconut oil on the alloxan-induced oxidative stress in the liver, kidneys and heart of diabetic rats. Food Funct 2019; 10: 2114-24. doi: 10.1039/ c9fo00107g

22. Carnevale R, Pastori D, Nocella C, Cammisotto V, Bartimoccia S, Novo M, et al. Gut-derived lipopolysaccharides increase post-prandial oxidative stress via Nox2 activation in patients with impaired fasting glucose tolerance: effect of extra-virgin olive oil. Eur J Nutr 2019; 58: 843-51. doi: 10.1007/ s00394-018-1718-x

23. Iredale JP. Models of liver fibrosis: exploring the dynamic nature of inflammation and repair in a solid organ. J Clin Invest 2007; 117: 539-48. doi: 10.1172/JCI30542

24. Seki E, Schwabe RF. Hepatic inflammation and fibrosis: functional links and key pathways. Hepatology 2015; 61: 1066-79. doi: 10.1002/hep. 27332

25. Choi I, Kang HS, Yang Y, Pyun KH. IL-6 induces hepatic inflammation and collagen synthesis in vivo. Clin Exp Immunol 1994; 95: 530-5. doi: 10.1111/j.1365-2249.1994.tb07031.x

26. Yin $\mathrm{M}$, Wheeler MD, Kono H, Bradford BU, Gallucci RM, Luster MI, et al. Essential role of tumor necrosis factor alpha in alcohol-induced liver injury in mice. Gastroenterology 1999; 117: 942-52. doi: 10.1016/s0016-5085(99)70354-9

27. Osawa Y, Hoshi M, Yasuda I, Saibara T, Moriwaki H, Kozawa O. Tumor necrosis factor-alpha promotes cholestasis-induced liver fibrosis in the mouse through tissue inhibitor of metalloproteinase-1 production in hepatic stellate cells. Plos One 2013; 8: e65251. doi: 10.1371/journal.pone.0065251

28. Connolly MK, Bedrosian AS, Mallen-St CJ, Mitchell AP, Ibrahim J, Stroud A, et al. In liver fibrosis, dendritic cells govern hepatic inflammation in mice via TNF-alpha. J Clin Invest 2009; 119: 3213-25. doi: 10.1172/JCI37581

29. Valente AJ, Rozek MM, Sprague EA, Schwartz CJ. Mechanisms in intimal monocyte-macrophage recruitment. A special role for monocyte chemotactic protein-1. Circulation 1992; 86: I20-5.

30. Lai SS, Fu X, Cheng Q, Yu ZH, Jiang EZ, Zhao DD, et al. HSC-specific knockdown of GGPPS alleviated CCl4-induced chronic liver fibrosis through mediating RhoA/Rock pathway. Am J Transl Res 2019; 11: 2382-92.

31. Cao F, Zhang Y, Li W, Shimizu K, Xie H, Zhang C. Mogroside IVE attenuates experimental liver fibrosis in mice and inhibits HSC activation through downregulating TLR4-mediated pathways. Int Immunopharmacol 2018; 55: 183-92. doi: 10.1016/j. intimp.2017.12.023

32. Han M, Liu X, Liu S, Su G, Fan X, Chen J, et al. 2,3,7,8-Tetrachlorodibenzo-p-dioxin (TCDD) induces hepatic stellate cell (HSC) activation and liver fibrosis in C57BL6 mouse via activating Akt and NF-kappaB signaling pathways. Toxicol Lett 2017; 273: 10-19. doi: 10.1016/j.toxlet.2017.03.013

33. Lewindon PJ, Pereira TN, Hoskins AC, Bridle KR, Williamson RM, Shepherd RW, et al. The role of hepatic stellate cells and transforming growth factor-beta(1) in cystic fibrosis liver disease. Am J Pathol 2002; 160: 1705-15. doi: 10.1016/ s0002-9440(10)61117-0

34. Peng X, Dai C, Liu Q, Li J, Qiu J. Curcumin attenuates on carbon tetrachloride-induced acute liver injury in mice via modulation of the Nrf2/HO-1 and TGF-beta1/Smad3 pathway. Molecules 2018; 23. doi: 10.3390/molecules23010215

35. Seki E, De Minicis S, Österreicher CH, Kluwe J, Osawa Y, Brenner DA, et al. TLR4 enhances TGF- $\beta$ signaling and hepatic fibrosis. Nat Med 2007; 13: 1324-32. doi: 10.1038/ nm1663

\section{* Xundi $\mathrm{Xu}$}

Hunan Provincial Key Laboratory of Hepatobiliary Disease

Research

Division of Hepato-Biliary-Pancreatic Surgery

Department of Surgery

The Second Xiangya Hospital

Central South University

Changsha 410000

Hunan, China

Email:xuxundi@csu.edu.cn 